Original Paper http://ajol.info/index.php/ijbes http://indexmedicus.afro.who.int

\title{
Effet de l'inoculation mycorhizienne avec des souches des champignons mycorhiziens arbusculaires sur la croissance de Mucuna pruriens (L.) DC en condition contrôlée
}

\author{
Hadou HARO ${ }^{*}$, Kadidia SEMDE ${ }^{1}$, Kadidiata BAHADIO ${ }^{2}$ et Kadidia B. SANON ${ }^{1}$ \\ ${ }^{1}$ Laboratoire de Microbiologie, INERA/DEF BP 7047 Ouagadougou 03, Burkina Faso. \\ ${ }^{2}$ Institut du Développement Rural (IDR), Université NAZI BONI 01 BP 1091 Bobo-Dioulasso 01, Burkina Faso. \\ *Auteur correspondant ; E-mail: harohadou@yahoo.fr, Tel :(00226)76627083
}

\section{RESUME}

Au Burkina Faso, l'élevage occupe plus de $80 \%$ des ménages ruraux et constitue le troisième produit d'exportation après l'or et le coton. De type extensif, cet élevage est basé essentiellement sur l'exploitation des ressources naturelles. Ainsi, il doit s'adapter aux grandes variations saisonnières et interannuelles des ressources en biomasse végétale et en eau. Or les aléas climatiques, l'étendue et la qualité des pâturages, de même que les contraintes rendent l'activité pastorale souvent précaire. C'est ainsi que cette étude a été initiée dans le but de contribuer à améliorer la production fourragère. Dans cette étude, Mucuna pruriens a été cultivé en serre et inoculé avec deux inocula de champignons mycorhiziens arbusculaires. Les paramètres de croissance ont été mesurés à 30 et 60 jours après semis. La biomasse aérienne, racinaire et totale a été évaluée à 60 jours après semis. Les résultats montrent une amélioration de la croissance en hauteur du mucuna de $225,76 \%$, de la biomasse aérienne de $56,79 \%$, la biomasse racinaire de $70 \%$ et la biomasse totale de $61,16 \%$ par rapport au témoin non inoculé. Cette étude a montré des résultats intéressants et mérite d'être approfondie par des essais in situ tout en étendant l'étude sur les inoculations rhizobiennes.

(C) 2020 International Formulae Group. All rights reserved.

Mots clés : Mucuna, inoculation mycorhizienne, champignons mycorhiziens arbusculaires.

\section{Effect of mycorrhizal inoculation with arbuscular mycorrhizal fungi strains on Mucuna pruriens (L.) DC growth under controlled condition}

\begin{abstract}
In Burkina Faso, breeding occupies more than $80 \%$ of rural households and is the third export product after gold and cotton. Extensive type, this breeding is based essentially on the exploitation of natural resources. Thus, it must adapt to large seasonal and interannual variations in plant biomass and water resources. Climatic hazards, the extent and quality of pastures, as well as constraints make pastoral activity often precarious. Therefore, this study was initiated with the aim of helping to improve forage production. In this study, Mucuna pruriens was grown in a greenhouse and inoculated with two inocula of arbuscular mycorrhizal fungi. The growth
\end{abstract}


parameters were measured at 30 and 60 days after sowing. Shoot, root and total biomass was evaluated at 60 days after sowing. The results show an improvement in the height growth of mucuna by $225.76 \%$, shoot biomass by $56.79 \%$, root biomass by $70 \%$ and total biomass by $61.16 \%$ compared to the control. This study showed interesting results and deserves to be deepened by in situ tests while extending the study of rhizobial inoculations. (C) 2020 International Formulae Group. All rights reserved.

Keywords: Mucuna, mycorrhizal inoculation, arbuscular mycorrhizal fungi.

\section{INTRODUCTION}

Au Burkina Faso, l'élevage occupe une place importante dans l'économie du pays ; il contribue pour $18 \%$ à la formation du produit intérieur brut (PIB) et constitue une source de revenus pour environ $80 \%$ de la population (Kiema et al., 2014). Dans ce pays, l'élevage est de type extensif basé essentiellement sur l'exploitation des ressources naturelles. Les pratiques traditionnelles actuelles ne permettent plus de répondre efficacement aux problèmes d'alimentation et d'abreuvement des animaux or l'alimentation constitue l'un des facteurs limitant le plus important de la production animale. La majeure partie des ressources alimentaires provient des pâturages naturels qui, même si elles sont susceptibles d'amélioration, elles restent néanmoins limitées en étendue du fait de l'extension des terres cultivées par les pratiques agricoles extensives. Cette forme d'élevage doit s'adapter aux grandes variations saisonnières et interannuelles des ressources en biomasse végétale et en eau. Cependant au Burkina Faso, les aléas climatiques, l'étendue et la qualité des pâturages, de même que les contraintes sanitaires et économiques, rendent l'activité pastorale souvent précaire. Or, l'amélioration des productions animales requiert en plus d'un bon état de santé, des ressources alimentaires assez importantes en quantité et en qualité. Ainsi, il devient nécessaire de développer des méthodes permettant d'améliorer la quantité et la qualité de la biomasse végétale produite. De ce fait, le mucuna [Mucuna pruriens (L.) DC], une légumineuse héliophile, thermophile, à croissance vigoureuse, à port rampant et à but multiples (Aklamavo et Mensah, 1997) apparait comme une alternative pouvant contribuer à améliorer la production fourragère. Cependant, cinq poches de sécheresse sont survenues sur une période de 36 ans (1981-2017), une baisse de la productivité des terres et une dégradation de la biomasse végétale. Entre 1995 et 2015, l'occupation des terres a été en défaveur des zones de pâture (diminution de 13,08\%) (Sieza et al., 2019). La baisse de la productivité des terres serait liée à l'appauvrissement croissant des sols en éléments minéraux majoritairement l'azote et le phosphore. Or il est bien établi que le mucuna forme une symbiose avec les champignons mycorhiziens arbusculaires (Tchiegang-Megueni et al., 2000; Jemo et al., 2007). De plus, l'amélioration de l'absorption des éléments minéraux (le phosphore et l'azote), de la nutrition et de la croissance des plantes associées aux mycorhizes a été largement étudiée (Boureima et al., 2007; AtulNayyar et al., 2009; Haro et al., 2012; Diatta et al., 2013; Haougui et al., 2013; Haro et al., 2015; Manga et al., 2017). Aussi, il a été démontré que la croissance et la production des plantes sont améliorer par la symbiose mycorhizienne même lorsque ces plantes poussent sur des sols relativement pauvres en éléments minéraux (Haro, 2016; Haro et Sanon, 2016; Haro et al., 2016a; Haro et al., 2016c; Haro et al., 2016b; Haro et al., 2016d; Haro et al., 2017). L'exploitation de cette symbiose serait une possibilité pour améliorer la productivité de biomasse de Mucuna pruriens et c'est ainsi que nous nous sommes intéressés à la symbiose mycorhizienne du mucuna avec pour objectif d'évaluer sa réponse à l'inoculation mycorhizienne avec les champignons mycorhiziens arbusculaires (CMA) en condition contrôlée. 


\section{MATERIEL ET METHODES}

\section{Matériel}

Matériel végétal

Pour évaluer l'effet de l'inoculation mycorhiienne sur le mucuna, les graines de Mucuna pruriens ont été utilisées. Ces graines ont été obtenues au Centre de Recherche Environnementales Agricoles et de Formation (CREAF) de Kamboinsé (Burkina Faso).

\section{Matériel fongique}

Le matériel fongique était composé de deux inocula de champignons mycorhiziens arbusculaires (CMA) dont un inoculum mixte local efficient isolé de la rhizosphère du niébé cultivé au Burkina Faso [Scutellospora sp., Gigaspora sp., Glomus sp. (Yac 2 mix)] (Haro, 2016) et un inoculum fongique de la collection du Laboratoire Commun de Microbiologie (LCM) du Sénégal [Glomus aggregatum (Ga)].

\section{Substrat de culture}

Le substrat de culture était un sol stérilisé $\left(1 \mathrm{~h}\right.$ à $\left.120^{\circ} \mathrm{C}\right)$ de Ouagadougou dont les caractéristiques physico-chimiques sont : argile : $3,92 \%$; limons totaux : $5,88 \%$; sable totaux : $90,2 \%$; matière organique totale : $0,331 \%$; carbone total : $0,192 \%$; azote total : $0,016 \% ; \mathrm{C} / \mathrm{N}: 12$; phosphore total : $172,52 \mathrm{ppm}$; phosphore assimilable : 1,74 ppm ; pH H2O (p/v : 1/2,5), 6,44.

\section{Méthode}

\section{Traitement des graines}

Les graines de mucuna ont été aseptisées par trempage dans l'éthanol $96^{\circ}$ pendant 3 minutes, rincées abondamment à l'eau distillée stérile puis dans une solution d'hypochlorite de calcium $\left(\mathrm{CaCl}_{2} \mathrm{O}_{2}\right.$ à $3,3 \%$; $\mathrm{p} / \mathrm{v})$ pendant $3 \mathrm{~min}$ et rincées abondamment à l'eau distillée stérile. Ces graines sont ensuite semées à raison de 2 par pot.

\section{Mise en place de l'essai}

L'essai avait consisté à cultiver le mucuna dans des pots de 2 litres contenant $2000 \mathrm{~g}$ de substrat de culture. Le dispositif expérimental utilisé est de type randomisé simple. Ce dispositif était composé de 3 traitements [deux traitements inoculés (Yac 2 mix et $\mathrm{Ga}$ ) et un témoin non inoculé)] et chaque traitement a été répété 5 fois. Le mucuna a été semé à raison de 2 graines par pot et le démariage est intervenu à la deuxième semaine après semis de sorte à laisser un plant par pot. Cette culture a duré 60 jours.

Les inocula mycorhiziens ont été apportés au moment des semis. L'inoculum mycorhizien était composé d'un mélange de spores, de propagules fongiques, de fragments de racines mycorhizées et de sol. L'inoculation mycorhizienne a consisté à l'apport de l'inoculum mycorhizien à raison de $10 \mathrm{~g}$ par pot pendant les semis.

Pour estimer la réponse du mucuna à l'inoculation mycorhizienne, la hauteur, le diamètre au collet, le taux de croissance relative en hauteur et le taux de croissance relative du diamètre au collet ont été mesurés au $30^{\mathrm{e}}$ et au $60^{\mathrm{e}}$ jour après semis. Les biomasses aérienne et racinaire ainsi que la fréquence et l'intensité de mycorhization ont été mesurées à la fin de l'essai (60 jour après semis).

Mesure de la hauteur, du diamètre au collet, du taux de croissance relative en hauteur et du taux de croissance relative du diamètre au collet

La hauteur a été mesurée à l'aide d'une règle graduée à 30 et 60 jours après semis. Le taux de croissance relative en hauteur (TCRh) a été calculé selon la formule suivante :

$\mathrm{TCRh}=(\mathrm{Hf}-\mathrm{Hi}) / \mathrm{Hi}$ avec $\mathrm{H}:$ hauteur, $\mathrm{i}$ : initiale, $f$ : finale.

Le diamètre au collet a été mesuré à l'aide d'un pied à coulisse au niveau de la zone de séparation entre le système racinaire et la partie aérienne à 30 et 60 jours après semis. Le taux de croissance relative du diamètre au collet (TCRdc) a été calculé selon la formule suivante :

TCRdc $=($ Dcf - Dci $) /$ Dci, avec Dc : Diamètre au collet, $\mathrm{i}$ : initiale et $\mathrm{f}: \mathrm{f}$ : finale.

Mesure de la biomasse aérienne, racinaire et totale

A 60 jours après semis, chaque plant a été dépoté soigneusement de façon à récupérer la partie aérienne et toutes les racines des plants. Toutes ces parties ont été séchées à l'étuve à $70^{\circ} \mathrm{C}$ pendant 72 heures pour la mesure de la biomasse aérienne, racinaire et totale. Après la mesure de la biomasse, les racines ont été utilisées pour l'étude de l'infection mycorhizienne. 


\section{Calcul du taux d'amélioration}

Le taux d'amélioration de

l'inoculation mycorhizienne a été calculé selon la formule suivante:

$$
A=\frac{(V m-V t)}{V t} x 100 \text { avec A : taux }
$$

d'amélioration (\%), Vm : valeur du traitement inoculé ayant obtenu la valeur maximale et statistiquement différente du témoin, Vt: valeur du traitement témoin.

\section{Mise en évidence de l'infection mycorhizienne}

La mise en évidence de l'infection endomycorhizienne a été réalisée par la coloration des racines fines des plants selon la méthode de Phillips et Hayman (1970) et l'observation au microscope photonique. Les racines fines des plants ont été découpées en fragments d'un centimètre et ont été colorées suivant la méthode décrite par Phillips et Hayman (1970). Les fragments colorés ont été montés entre lames et lamelles dans du glycérol. La fréquence et l'intensité de mycorhization ont été évaluées au microscope optique au grossissement 400 en utilisant la méthode de Trouvelot et al. (1986).

\section{Analyse statistique}

Toutes les données collectées pour les différents paramètres ont été soumises à une analyse de variance (ANOVA) avec le logiciel statistique XLSTAT 2018 et les moyennes des variables ont été comparées en utilisant le test de Newman Keuls au seuil de probabilité $\mathrm{p}=5$ $\%$.

\section{RESULTATS}

\section{Evaluation de la fréquence et de l'intensité de mycorhization}

Le Tableau 1 montre la fréquence et l'intensité de mycorhization du mucuna. La fréquence de mycorhization est assez élevée (90\% pour l'inoculum Yac 2 mix) alors que l'intensité de mycorhization reste faible $(28,75 \%)$ pour l'inoculum Yac $2 \mathrm{mix})$. Les analyses statistiques montrent des différences significatives entre les différents traitements. Cependant, les racines des traitements témoins ne sont pas mycorhizées.
Mesure de la hauteur, du taux de croissance relative en hauteur, du diamètre au collet et du taux de croissance relative du diamètre au collet du mucuna

Le Tableau 2 présente les résultats des mesures de la hauteur, du taux de croissance relative en hauteur, du diamètre au collet et du taux de croissance relative du diamètre au collet du mucuna inoculé avec des champignons mycorhiziens arbusculaires (CMA) indigènes. Les résultats montrent que la hauteur des plants varie en fonction de l'inoculum utilisé et au fil du temps. A 30 jours après semis, l'analyse statistique ne montre aucune différence significative $(\mathrm{P}>0,05)$ entre les différents traitements ni pour la hauteur ni pour le diamètre au collet. A 60 jours après semis, les différences apparaissent entre les différents traitements pour la hauteur et les valeurs les plus élevées s'obtiennent pour l'inoculation avec l'inoculum Yac $2 \mathrm{mix}$ $(239,67 \mathrm{~cm})$. Cet inoculum permet d'améliorer la croissance en hauteur du mucuna $170,51 \%$ comparée aux témoins non inoculés. Cependant, les analyses statistiques ne montrent aucune différence significative $(\mathrm{P}>0,05)$ entre les différents traitements, pour le taux de croissance relative en hauteur, pour taux de croissance relative du diamètre au collet ainsi que pour la croissance en diamètre au collet. De façon générale l'inoculum Yac 2 mix permet d'améliorer la croissance du mucuna.

\section{Evaluation de la biomasse aérienne, racinaire et totale}

Les résultats sur la biomasse aérienne, racinaire et totale sont présentés dans le Tableau 3. Ces résultats sont variables en fonction des traitements. Les analyses statistiques montrent des différences significatives entre les différents traitements. Les valeurs les plus élevées s'obtiennent avec l'inoculum Yac 2 mix (5,08 g pour la biomasse aérienne, 2,72 $\mathrm{g}$ pour la biomasse racinaire et $7,8 \mathrm{~g}$ pour la biomasse totale). Cet inoculum permet d'améliorer la biomasse aérienne de $56,79 \%$, la biomasse racinaire de $70 \%$ et la biomasse totale de $61,16 \%$ par rapport au témoin non inoculé. 
Tableau 1 : Fréquence et intensité de mycorhization du sésame inoculé avec deux souches de mycorhize indigène [complexe mycorhiziens (Yac 2 mix) et Glomus sp. (Ga)].

\begin{tabular}{lcc}
\hline Traitements & $\begin{array}{c}\text { Fréquence de } \\
\text { mycorhization }(\%)\end{array}$ & Intensité de mycorhization (\%) \\
\hline Yac 2 mix & $90 \pm 5,26^{\mathrm{a}}$ & $28,75 \pm 3,34^{\mathrm{a}}$ \\
$\mathrm{Ga}$ & $71 \pm 3,33^{\mathrm{b}}$ & $4,75 \pm 0,75^{\mathrm{b}}$ \\
Témoin & $0^{\mathrm{c}}$ & $0^{\mathrm{c}}$ \\
Niveau de signification & $<0,0001$ & $<0,0001$ \\
\hline
\end{tabular}

Témoin = non inoculé.

Pour la même colonne (fréquence et intensité de mycorhization), les valeurs qui ont en commun une même lettre ne sont pas différentes significativement selon le test de Newman-Keuls au seuil de 5\%.

Tableau 2 : Hauteur, taux de croissance relative en hauteur, diamètre au collet et taux de croissance relative du diamètre au collet du sésame inoculé avec deux souches de mycorhize indigène [complexe mycorhiziens (Yac 2 mix) et Glomus sp. (Ga)].

\begin{tabular}{|c|c|c|c|c|c|c|}
\hline Traitements & $\begin{array}{l}\text { Hauteur } 1 \\
(\mathrm{~cm})\end{array}$ & $\begin{array}{l}\text { Hauteur } 2 \\
\text { (cm) }\end{array}$ & TCRh (\%) & $\begin{array}{l}\text { Diamètre } \\
1(\mathrm{~mm})\end{array}$ & $\begin{array}{l}\text { Diamètre } \\
2(\mathrm{~mm})\end{array}$ & $\begin{array}{l}\text { TCRdc } \\
(\%)\end{array}$ \\
\hline Yac 2 mix & $66,17 \pm 10,67^{a}$ & $239,67 \pm 23,88^{\mathrm{a}}$ & $2,81 \pm 0,53^{\mathrm{a}}$ & $2,45 \pm 0,12^{\mathrm{a}}$ & $1,81 \pm 0,22^{\mathrm{a}}$ & $0,4 \pm 0,22^{\mathrm{a}}$ \\
\hline $\mathrm{Ga}$ & $97 \pm 10,78^{a}$ & $182 \pm 20,46^{\mathrm{ab}}$ & $1,64 \pm 0,21^{\mathrm{a}}$ & $2,81 \pm 0,26^{\mathrm{a}}$ & $1,64 \pm 0,14^{\mathrm{a}}$ & $0,71 \pm 0,26^{\mathrm{a}}$ \\
\hline Témoin & $31,7 \pm 10,87^{\mathrm{a}}$ & $88,6 \pm 12,27^{\mathrm{b}}$ & $1,69 \pm 1,08^{\mathrm{a}}$ & $2,39 \pm 1,2^{\mathrm{a}}$ & $1,69 \pm 0,1^{\mathrm{a}}$ & $0,44 \pm 0,1^{\mathrm{a}}$ \\
\hline $\begin{array}{l}\text { Niveau de } \\
\text { signification }\end{array}$ & NS & $<0,001$ & NS & NS & NS & NS \\
\hline $\begin{array}{l}\text { Hauteur } 1 \text { et } \\
\text { Diamètre } 1 \text { et } \\
\text { au collet. Tén } \\
\text { Pour la mêm } \\
\text { diamètre au c } \\
\text { Newman-Kè }\end{array}$ & $\begin{array}{l}\text { hauteur mesurée } 1 \\
\text { : diamètre mesuré } \\
\text { in = non inoculé. } \\
\text { olonne (hauteur, } t \\
\text { let), les valeurs qu } \\
\text { au seuil de } 5 \% \text {. }\end{array}$ & $\begin{array}{l}\text { ctivement à } 30 \text { et } \\
\text { ectivement à } 30 \text { et } \\
\text { non significatif. } \\
\text { de croissance rela } \\
\text { en commun une }\end{array}$ & $\begin{array}{l}\text { ours après } \\
\text { urs après s } \\
\text { en hauteur, } \\
\text { e lettre ne }\end{array}$ & $\begin{array}{l}\text { TCRh : tau } \\
\text { TCRdc : tau } \\
\text { etre au collc } \\
\text { as différent }\end{array}$ & $\begin{array}{l}\text { oissance rela } \\
\text { oissance rela } \\
\text { ux de croiss } \\
\text { ficativement }\end{array}$ & $\begin{array}{l}\text { en hauteur. } \\
\text { du diamètre } \\
\text { lon relative du } \\
\text { lest de }\end{array}$ \\
\hline
\end{tabular}

Tableau 3 : Biomasse aérienne, racinaire et totale du sésame inoculé avec deux souches de mycorhize indigène [complexe mycorhiziens (Yac 2 mix) et Glomus sp. (Ga)].

\begin{tabular}{lccc}
\hline Traitements & Biomasse aérienne (g) & Biomasse racinaire (g) & Biomasse totale (g) \\
\hline Yac 2 mix & $5,08 \pm 0,23^{\mathrm{a}}$ & $2,72 \pm 0,12^{\mathrm{a}}$ & $7,8 \pm 0,35^{\mathrm{a}}$ \\
$\mathrm{Ga}$ & $4,3 \pm 0,15^{\mathrm{b}}$ & $1,57 \pm 0,08^{\mathrm{b}}$ & $5,87 \pm 0,23^{\mathrm{b}}$ \\
Témoin & $3,24 \pm 0.06^{\mathrm{c}}$ & $1,6 \pm 0,09^{\mathrm{c}}$ & $4,84 \pm 0,07^{\mathrm{b}}$ \\
Niveau de signification & $<0,001$ & $<0,001$ & $<0,001$ \\
\hline
\end{tabular}

Témoin = non inoculé.

Pour la même colonne (biomasse aérienne, racinaire ou totale), les valeurs qui ont en commun une même lettre ne sont pas différentes significativement selon le test de Newman-Keuls au seuil de 5\%. 


\section{DISCUSSION}

Cette étude a été menée avec pour objectif d'évaluer l'effet de l'inoculation mycorhizienne de Mucuna pruriens en milieu contrôlé. Les résultats sur la mycorhization montrent que les racines du mucuna sont mycorhizées par les souches mycorhiziennes utilisées (Tableau 1). L'absence d'infection mycorhizienne sur les racines des témoins montre que les traitements sont exempts de toute contamination mycorhizienne. $\mathrm{La}$ stimulation de la croissance et de la biomasse entre les différents traitements comparés au témoin pourrait être attribuée à l'effet des champignons mycorhiziens arbusculaires inoculés.

Les résultats obtenus sur la croissance $\mathrm{du}$ mucuna montrent que l'inoculation améliore la croissance de cette plante (Tableaux 2 et 3 ). Les analyses statistiques sur la hauteur des plants mesurés à 30 jours après semis ne montrent aucune différence significative entre les plants inoculés et le témoin (Tableau 2). Ceci s'explique par le fait que le substrat utilisé contient des éléments nutritifs nécessaire et directement accessible aux racines des plants qui assurent convenablement leur nutrition. Par conséquent, ces plants ne trouvent pas de nécessité de passer par les CMA pour assurer leur nutrition minérale d'où l'inefficacité de l'inoculation mycorhizienne sur la croissance en hauteur et le diamètre au collet du mucuna à 30 jours après semis. Ces résultats corroborent ceux de Haro et al. (2012) qui ont montré que la plante ne trouvera pas de nécessité de former la symbiose mycorhizienne si les éléments nutritifs sont disponibles dans le milieu et directement accessible aux racines de la plante. Ceci explique également l'absence de différences significatives sur le taux de croissance relative en hauteur, le taux de croissance relative du diamètre au collet du mucuna. Cependant, les analyses statistiques sur la hauteur des plants mesurés à 60 jours après semis montrent des différences significatives entre les différents traitements (Tableau 2). Ceci s'explique par le fait que pendant la croissance des plants, les éléments nutritifs directement accessibles aux racines se sont épuisés au cours du temps. Ainsi, les racines deviennent incapables d'assurer convenablement la nutrition minérale de ces plants. C'est ainsi que les plants ont recours aux CMA pour assurer leur nutrition minérale. Ainsi, la symbiose mycorhizienne s'établit et se développe progressivement avec l'épuisement des éléments minéraux dans le sol d'où l'efficacité des souches mycorhiziennes dans l'amélioration de la croissance en hauteur des plants de mucuna au $60^{\mathrm{e}}$ jour après semis. Ces résultats sont en concordance avec ceux de Haro et al. (2012) qui ont montré que la symbiose mycorhizienne s'établit et se développe sur le niébé avec l'épuisement des éléments minéraux directement accessible aux racines dans le sol. Les valeurs les plus élevées s'obtiennent avec l'inoculum Yac 2 mix (Tableau 2). Ceci s'explique par l'important pouvoir d'absorption minérale de l'inoculum Yac 2 mix.

Pour la biomasse aérienne, racinaire et totale, l'inoculum Yac 2 mix a montré la meilleure efficacité (Tableau 3). Tout comme pour la hauteur mesurée au $60^{\mathrm{e}}$ jour après semis, l'efficacité de l'inoculum Yac 2 mix sur la stimulation de la production de biomasse pourrait s'expliquer par l'amélioration de la nutrition minérale du mucuna par cet inoculum. Ces résultats corroborent ceux de Jemo et al. (2007) qui ont trouvé que la production de biomasse aérienne et racinaire de Mucuna pruriens est significativement améliorée par l'inoculation mycorhizienne. Des résultats analogues sont trouvés par Haro et al. (2017) qui ont montré que l'inoculation mycorhizienne du niébé avec les inocula indigènes permet d'améliorer la production de biomasse et le rendement de la production graine de cette plante. Ces résultats sont en concordance avec ceux de Diop et al. (2013) qui ont montré que la production de la biomasse de Vigna est améliorée par l'inoculation mycorhizienne. Des résultats analogues ont également été obtenus pour le trèfle inoculé avec G. mosseae (Khaled et al., 2003), pour Acacia nilotica et Acacia senegal inoculées avec un complexe mycorhizien de 
souches indigènes (Laminou Manzo et al., 2009 ) et pour le Robinier inoculé à $R$. irregularis et G. versiforme (Zhu et al., 2014).

Il ressort que Yac 2 mix est la plus efficace de tous les inocula utilisés dans cette étude. Ceci pourrait s'expliquer par le fait que Yac 2 mix renferment deux genres de CMA dont les effets peuvent s'ajouter par rapport à Ga qui n'a qu'une seule espèce. Des résultats similaires ont été trouvés par Haro (2011) qui a montré les souches indigènes contenant au moins 2 genres ont des effets qui peuvent s'ajouter comparé aux inocula monospécifiques étant donné que les endomycorhizes n'entrent pas en compétition.

\section{Conclusion}

Cette étude avait pour objectif d'évaluer la réponse $\mathrm{du}$ mucuna à l'inoculation mycorhizienne. De ces résultats, il ressort que l'inoculation mycorhizienne avec l'inoculum indigne permet d'améliorer la croissance et la production de biomasse de Mucuna pruriens. Il apparait que l'effet de l'inoculation est variable en fonction de l'inoculum. En plus des mycorhizes, les plantes de mucuna vivent naturellement en symbiose avec les bactéries du sol (rhizobia). Il serait intéressant qu'une étude complémentaire s'intéresse à l'inoculation rhizobienne et aussi à la double inoculation mycorhizienne et rhizobienne afin d'évaluer l'effet de ces inoculations sur la croissance et la production de biomasse de Mucuna pruriens.

\section{CONFLIT D'INTERETS}

Les auteurs déclarent qu'ils n'ont aucun conflit d'intérêts.

\section{CONTRIBUTIONS DES AUTEURS}

HH a réalisé toutes les activités (conception, mise en place de l'essai, récolte et l'analyse des données et rédaction de ce manuscrit) de cette étude. KS et KB ont participé à la réalisation de la majorité des activités de cette étude. KBS a été mise en sa mémoire pour lui rende hommage pour tous les sacrifices consentis pour notre encadrement.

\section{REMERCIEMENTS}

Ce travail a bénéficié du soutien du Laboratoire Mixte International (LMI) «Intensification écologique des sols cultivés en Afrique de l'Ouest » (IESOL).

\section{REFERENCES}

Aklamavo M, Mensah GA. 1997. Quelques aspects de l'utilisation du Mucuna en milieu rural en République du Bénin. Bulletin de la Recherche Agronomique, 19: 34-46.

Atul-Nayyar A, Hamel C, Hanson K, Germida J. 2009. The arbuscular mycorrhizal symbiosis links $\mathrm{N}$ mineralization to plant demand. Mycorrhiza, 19(4): 239-246. DOI: $10.1007 / \mathrm{s} 00572-008-0215-0$

Boureima S, Diouf M, Diop TA, Diatta M, Leye EM, Ndiaye F, Seck D. 2007. Effects of arbuscular mycorrhizal inoculation on the growth and the development of sesame (Sesamum indicum L.). African Journal of Agricultural Research, 3(3): 234-238.

Diatta MB, Manzo OL, Diouf PRM, Diop T. 2013. Effets de l'inoculation mycorhizienne sur le sesame (Sesamum indicum L.) en conditions naturelles. International Journal of Biological and Chemical Sciences, 7(5): 2050-2057. DOI:

http://dx.doi.org/10.4314/ijbcs.v7i5.22

Diop I, Kane A, Krasova-Wade T, Sanon KB, Houngnandan P, Neyra M, Noba K. 2013. Impacts des conditions pédoclimatiques et du mode cultural sur la réponse du niébé (Vigna unguiculata L. Walp.) à l'inoculation endomycorhizienne avec Rhizophagus irregularis. Journal of Applied Biosciences, 69: 5465-5474. DOI: http://dx.doi.org/10.4314/jab.v69i0.9507 2

Haougui A, Souniabe PS, Doumma A, Adam T. 2013. Evolution des populations des champignons endomycorhiziens sur les adventices de quatre sites maraîchers de la région de Maradi au Niger. International Journal of Biological and 
Chemical Sciences, 7(2): 554-565. DOI: http://dx.doi.org/10.4314/ijbcs.v7i2.12

Haro H. 2011. Effet d'inoculums de champignons mycorhiziens arbusculaires sur la productivité du niébé Vigna unguiculata (L.) Walp. Mémoire de DEA, Université de Ouagadougou. p. 94.

Haro H. 2016. Optimisation des symbioses rhizobienne et mycorhizienne pour améliorer la productivité du niébé [Vigna unguiculata (L.) Walp.] au Burkina Faso. Mémoire de Doctorat, Université Ouaga 1 Professeur Joseph Ki-Zerbo. p. 241.

Haro H, Sanon KB 2016. Amélioration des rendements du niébé avec les microorganismes symbiotiques indigènes au Burkina Faso Colloque IAVAO/IESOL/LAPSE « Biodiversité et processus écologiques au service d'une agriculture durable en Afrique de l'Ouest ». Dakar (Sénégal).

Haro H, Sanon KB, Blagna F, Fofana B. 2016a. Effect of native arbuscular mycorrhiza fungi inocula on the growth of Cowpea [Vigna unguiculata (L.) Walp.] in three differents agro ecological zones in Burkina Faso. Journal of Applied Biosciences, 108: 10553-10560. DOI: http://dx.doi.org/10.4314/jab.v108i1.8

Haro H, Sanon KB, Diop I, Kane A, Dianda M, Houngnandan P, Neyra M, Traoré A. 2012. Réponse à l'inoculation mycorhizienne de quatre variétés de niébé [Vigna unguiculata (L.) Walp.] cultivées au Burkina Faso et au Sénégal. International Journal of Biological and Chemical Sciences, 6(5): 2097-2112. DOI: http://dx.doi.org/10.4314/ijbcs.v6i5.18

Haro H, Sanon KB, Krasova-Wade T, Kane A, N'Doye I, Traoré AS. 2015. Réponse à la double inoculation mycorhizienne et rhizobienne du niébé (variété, KVX3964-5-2D) cultivé au Burkina Faso. International Journal of Biological and Chemical Sciences, 9(3): 1485-1493. DOI:

http://dx.doi.org/10.4314/ijbcs.v9i3.31
Haro H, Sanon KB, Le Roux C, Duponnois R, Traoré AS. 2017. Improvement of cowpea productivity by rhizobial and mycorrhizal inoculation in Burkina Faso. Symbiosis: 1-14. DOI: 10.1007/s13199017-0478-3

Haro H, Sanon KB, Traoré AS 2016b. Amélioration de la production du niébé par des engrais biologique (inoculation rhizobienneet mycorhizienne). Symposium International sur la Science et la Technologie (SIST 2016) «Valorisation des résultats de recherche sur les filières : Bétail-Viande, Karité, Maïs, Mangue, Mil, Niébé, Oignon, Riz, Sorgho, Tomate, Volaille locale ». Ouagadougou (Burkina Faso).

Haro H, Sanon KB, Traoré AS. 2016c. Amélioration de la productivité du niébé par l'inoculation rhizobienne et mycorhizienne à Yakouta, Nord du Burkina Faso. Science et Technique, Sciences Naturelles et Agronomie, (2): 111-121.

Haro H, Sanon KB, Traoré AS 2016d. Amélioration de la productivité du niébé par l'inoculation rhizobienne et mycorhizienne à Yakouta, Nord du Burkina Faso. Symposium International sur la Science et la Technologie (SIST 2016) «Valorisation des résultats de recherche sur les filières : Bétail-Viande, Karité, Maïs, Mangue, Mil, Niébé, Oignon, Riz, Sorgho, Tomate, Volaille locale ». Ouagadougou (Burkina Faso).

Jemo M, Nolte C, Nwaga D 2007. Biomass production, $\mathrm{N}$ and $\mathrm{P}$ uptake of Mucuna after Bradyrhizobia and Arbuscular mycorrhizal fungi inoculation, and Papplication on acid soil of Southern Cameroon. Dordrecht: Springer Netherlands. 855-864.

Khaled LB, Gomez AM, Quarraqi El M, Oihabi A. 2003. Réponses physiologiques et biochimiques du trèfle (Trifolium alexandinum. L.) à la double association mycorhizes rhizobiums sous une contrainte saline. Agronomie, 23: 571-580. DOI: 10.1051/agro:2003037 
Kiema A, Tontibomma G, Zampaligré N. 2014. Transhumance et gestion des ressources naturelles au Sahel : contraintes et perspectives face aux mutations des systèmes de productions pastorales. VertigO - la revue électronique en sciences de l'environnement, 14(3). DOI: https://doi.org/10.4000/vertigo.15404.

Laminou Manzo O, Ibrahim D, Campanella B, Paul R. 2009. Effets de l'inoculation mycorhizienne du substrat sur la croissance et la résistance au stress hydrique de cinq espèces fixatrices de dunes : Acacia raddiana Savi ; Acacia nilotica (L.) Willd. Ex Del. var. adansonii ; Acacia senegal (L.) Willd ; Prosopis chilensis Stunz. et Bauhinia rufescens Lam. Geo-Eco-Trop, 33: 115-124.

Manga A, Ndiaye F, Diop TA. 2017. Le champignon arbusculaire Glomus aggregatum améliore la nutrition minérale de Acacia seyal soumis au stress salin progressif. International Journal of Biological and Chemical Sciences, 11(5): 2352-2365.

DOI: http://dx.doi.org/10.4314/ijbcs.v7i5.10.

Phillips JM, Hayman DS. 1970. Improved procedures for clearing roots and staining parasitic and vesicular-arbuscular mycorrhizal fungi for rapid assessment of infection. Transactions of the British Mycological Society, 55(1): 158-161.

Sieza Y, Gomgnimbou APK, Serme I, Belem A. 2019. Etude de la variabilité climatique sur la dynamique d'occupation et d'utilisation des terres à des fins agropastorales dans la zone sud-soudanienne du Burkina Faso. International Journal of Biological and Chemical Sciences, 13(4): 1980-1994.

DOI: https://dx.doi.org/10.4314/ijbcs.v13i4.6.

Tchiegang-Megueni C, Nwaga D, Mapongmetsem PM, Lirawa P 2000. Effects of rhizobial and mycorrhizal biofertilizers on Mucuna pruriens (L.) DC (Papilionaceae) in greenhouse. Annales de l'Institut National de la Recherche Agronomique de Tunisie: Institut National de la Recherche Agronomique de Tunisie (INRAT). 197210.

Trouvelot A, Kough JL, Gianinazzi-Pearson V, Gianinazzi S. 1986. Mesure du taux de mycorhization VA d'un système radiculaire. Recherche de méthodes d'estimation ayant une signification fonctionnelle. Mycorrhizae : physiology and genetics; 217-221.

Zhu XQ, Wang CY, Chen H, Tang M. 2014. Effects of arbuscular mycorrhizal fungi on photosynthesis, carbon content, and calorific value of black locust seedlings. Photosynthetica, 52(2): 247-252. DOI: 10.1007/s11099-014-0031-z. 